\title{
Analysis of mRNA expression and DNA methylation level of RAC1 gene encoding focal adhesion molecule in endometrial and peritoneal endometriosis
}

\author{
Irwina Eka Deraya ${ }^{\mathrm{a}}$, Andon Hestiantoro ${ }^{\mathrm{b}, \mathrm{c}}$, Raden Muharam ${ }^{\mathrm{b}, \mathrm{c}}$, Muhammad Lucky Marwali ${ }^{\mathrm{d}}$, Agus Surur \\ As'adi $^{\mathrm{d}}$, Darmawi ${ }^{\mathrm{e}}$, Achmad Kemal Harzif ${ }^{\mathrm{c}}$, Gita Pratama ${ }^{\mathrm{b}, \mathrm{c}}$, Ocktariyana ${ }^{\mathrm{f}, \mathrm{g}}$, Annisah Zahrah ${ }^{\mathrm{a}}$, \\ Asmarinah $^{\mathrm{b}, \mathrm{h}^{*}}$ \\ ${ }^{a}$ Master Programme in Biomedical Sciences, Faculty of Medicine, Universitas Indonesia, Salemba, Jakarta, Indonesia \\ ${ }^{b}$ Human Reproductive, Infertility and Family Planning Cluster, IMERI, Faculty of Medicine, Universitas Indonesia, Salemba, Jakarta, \\ Indonesia \\ 'Department of Obstetrics and Gynecology, Faculty of Medicine, Universitas Indonesia, Salemba, Jakarta, Indonesia \\ ${ }^{d}$ Endometriosis Centre, Fatmawati General Hospital, Fatmawati, South Jakarta,Jakarta, Indonesia \\ ${ }^{e}$ Department of Histology, Faculty of Medicine, University of Riau, Sail, Pekanbaru, Indonesia \\ ${ }^{f}$ Doctoral Program in Biomedical Sciences, Faculty of Medicine, Universitas Indonesia \\ ${ }^{8}$ Ministry of Health, Health Polytechnic, Palembang, Indonesia \\ ${ }^{b}$ Department of Medical Biology, Faculty of Medicine, Universitas Indonesia, Salemba, Jakarta, Indonesia
}

Received 15th November 2019 / Accepted 24th February 2020

\begin{abstract}
Focal adhesion molecules involve in cellular migration, attachment, and play a role in endometriosis pathomechanisms. Recent studies showed that the expression of RAC1, a gene encoded focal adhesion molecule, was predominantly found in endometriosis. As gene expression may be regulated by DNA methylation. Therefore, this study aimed to analyze promoter methylation level of RAC1 gene and mRNA expression in endometrial and peritoneal endometriosis tissues. This study using 20 endometrial and 9 peritoneal tissues from the same patients and 20 normal endometrial. The DNA and RNA from samples were isolated, DNA was converted using sodium bisulfite and amplified using Methyl Specific Polymerase Chain Reaction (MSP) method. The methylation level was determined by the intensity measurement of the bands that arose in gel electrophoresis using Image J software, whereas mRNA expression level was measured by Reverse Transcription-quantitative PCR (RT-qPCR) method. The mRNA expression level of RAC1 gene in peritoneal endometriosis increased compared to normal endometrium, as well as compared to endometrial endometriosis, but there was no significant difference in endometrial endometriosis compared to normal. Promoter hypermethylation level of RAC1 gene in peritoneal endometriosis was significantly different compared to normal endometrium, however not significant to endometrial endometriosis. Methylation level of its gene in endometrial endometriosis shown no significant difference compared to normal. There was association between promoter hypermethylation level and its mRNA expression in endometrial endometriosis $(\mathrm{R}=0.014 ; \mathrm{p}=0.952)$. The elevation of mRNA expression of RAC1 gene plays a role in endometrial cell migration to peritoneum, and associated with promoter hypermethylation level of its gene.
\end{abstract}

Keywords: DNA methylation, focal adhesion, peritoneal, RAC1

*Author for correspondence: Asmarinah, Human Reproductive, Infertility and Family Planning Cluster, IMERI, Faculty of Medicine, Universitas Indonesia, Salemba, Jakarta, Indonesia. Email - asmarinah.si@gmail.com 


\section{INTRODUCTION}

Endometriosis often occurs in 6 to $10 \%$ women during their reproductive age. Endometriosis is defined as the presence of endometrial-like tissue located outside the uterine cavity that frequently found in ovaries and peritoneal (Sourial et al., 2014; Barcz et al., 2012). Common symptoms that may appear including dysmenorrhea, dyspareunia, and infertility. The widely accepted theory to explain endometriosis was Retrograde Menstruation containing cellular debris from menstrual endometrium and become a source for ectopic lesion (Soo et al., 2017).

The development of lesion may be affected by immune systems, apoptosis resistance, growth factors, and adhesion molecules (Aznaurova et al., 2014). Clusters of cell adhesion molecules such as integrins trigger cell attachment to a various extracellular matrix including fibronectin, laminin, and vitronectin drives cellular migration through interaction with the downstream regulation such as RAC1 (Burney et al., 2012; Jiang et al., 2012).

In recent years, extensive investigation of genes that may have contribution and association with endometriosis pathomechanisms has been conducted using genome-wide association studies (GWAS) (Liu and Zhao et al., 2016). Ping et al. (2016) reported that RAC1 mRNA expression, a gene encoded one of focal adhesion molecules, was found increase predominantly in endometrium women with endometriosis. RAC1 is a member of the Rho GTPase family, which includes RHO, RAC1, and CDC42 (Bid et al., 2013).

It has been known that focal adhesions act as a bridge between the cells and the extracellular matrix through integrins as a transmembrane protein that initiate adhesion and regulate assembly of actin cytoskeleton. Consequently, induced protrusion of the leading cells formed filopodia and lamellipodia for cell migration (Nagano et al., 2012; Haller, 2012). RAC1 is responsible for the process of malignancy transformation, angiogenesis, invasion, and metastasis (Bid et al., 2013). In addition, Liu and Zhao et al. (2016) has been identified that RAC1 increased its expression and suggested that has a role in the pathogenesis of endometriosis.
The increasing of the expression in genes can be caused by genetics or epigenetics modification. Epigenetics is combination between genetic and environmental factors that contribute to the disease etiology (Acosta \& Fallin 2016). One of epigenetics modifications that highly contributed to the pathogenesis of endometriosis was DNA methylation, regulates either silencing or activation of gene expressions through hypo or hypermethylation (Koukora et al., 2014; Schübeler, 2015; Gupta et al., 2015).

RAC1 expression has been linked to in diverse phenomenon including cellular transformations, migrations and may relate to pathogenesis of endometriosis. Unfortunately, the evidences of epigenetics modifications such as promoter methylation and its relations to affect mRNA expression of RAC1 in peritoneal and endometrial endometriosis are still limited. Therefore, this study aimed to analyze relation of DNA methylation of RAC1 promoter to mRNA expression level in endometrial and peritoneal endometriosis. Thus, it may explain contributions of methylation level of RAC1 gene as focal adhesion gene in endometriosis pathomechanisms.

\section{MATERIALS AND METHODS}

\section{Sample preparation}

This cross-sectional study using 20 patients with endometriosis and 20 women as a normal group who underwent in vitro fertilization program with the range of the age in each group was $20-40$ years old. The samples were 20 endometrial tissues and 9 peritoneal tissues were taken from the same patient and 20 normal endometrial tissues. Sample collection was conducted according to the protocol of the World Endometriosis Research Foundation (Fassbender et al., 2014).

Samples were collected in the Department of Obstetrics and Gynecology, Faculty of Medicine, University of Indonesia-National Center General Hospital Dr. Cipto Mangunkusumo Jakarta and Endometriosis Centre, Fatmawati Hospital Jakarta. Ethical approval was obtained from 
Ethics Committee Faculty of Medicine Universitas Indonesia with protocol number 1901-0012. This research was conducted at Molecular Biology Laboratory, Integrated Laboratory, and Human Reproduction Cluster, IMERI from October 2018 to February 2019.

\section{Total RNA extraction}

Total RNA extraction was performed using Quick-RNA ${ }^{\mathrm{TM}}$ MiniPrep (Zymo, USA) according to the manufacture procedures.

\section{cDNA synthesis and $m R N A$ analysis}

The cDNA synthesis was performed from total RNA using Rever'Tra Ace ${ }^{\circledR}$ qPCR RT Master Mix with gDNA Remover (Toyobo, Japan). The cDNA samples were then amplified by q-RT PCR using THUNDERBIRD ${ }^{\mathrm{TM}}$ SYBR $^{\circledR}$ qPCR Mix (Toyobo, Japan). The total volume for each sample was $6 \mu \mathrm{L}$.

cDNA samples were amplified by q-RT PCR using THUNDERBIRD ${ }^{\text {TM }}$ SYBR $^{\circledR}$ qPCR Mix (Toyobo, Japan). RAC1 Primers for RT-PCR were designed by Primer Quest Tool and the primers were ordered from Integrated DNA Technologies (Integrated DNA Technologies, USA) Inc. Forward primer was GCTAAGGAGATTGGTG CTGTAA and Reverse was TCGGATCGCTTCG TCAAAC. Relative quantification of RAC1 mRNA expression using Livak's method was performed (Dorak 2006).

\section{Total DNA extraction}

The DNA from tissue samples was extracted using gSYNCTM DNA Extraction kit (Geneaid, Taiwan) according to the protocol.

\section{Promoter methylation analysis}

This promoter methylation analysis was following to a previous study that conducted by Darmawi et al. (2018). The extracted DNA samples were converted by sodium bisulfite using Epitect Bisulfite Kit (Qiagen, Germany). The total volume was $140 \mu \mathrm{L}$. The bisulfite-converted DNA was amplified by the methylation-specific polymerase chain reaction (MSP). Amplification of methylated and unmethylated primers were performed using MSP method. Primers were designed by MethPrimer program (http://www.urogene.org/cgi-bin/methprimer/ methprimer) and the primers were ordered from (Integrated DNA Technologies, USA) Inc. MSP was also used to the positive control samples (EpiTect Methylated Control DNA (human), Qiagen, Germany).

Methylated primers were Forward-M: AAGGGATTTGTAGGTTCGGTTAC; ReverseM: CCGAAATT'TCTCTAAACTTCGAA and unmethylated primer were Forward-U: AAGGGA T'TTGTAGGTT'TGGT'TATG; Reverse-U: CTC CCAAAATTTCTCTAAACTTCAA. The size of methylation amplicon was $129 \mathrm{bp}$, whereas an unmethylation was $132 \mathrm{bp}$. MSP product was visualized using 3\% gel electrophoresis and the band intensity was measured using ImageJ program. The level of promoter methylation of RAC1 gene was determined by comparing band intensity of methylated product to total band intensity from methylated and unmethylated band product.

\section{Statistical analysis}

mRNA expression level was analyzed using independent T-test and Mann-Whitney while promoter methylation level using Mann-Whitney test in endometrial, peritoneal endometriosis, and normal endometrium. Correlation between promoter methylation level and mRNA expression level in endometrial endometriosis using Spearman test

\section{RESULTS AND DISCUSSION}

\section{The mRNA expression level of RAC1 gene}

In this study, we determined the level of expression in RAC1 gene and compared the expression between normal endometrium, endometrial and peritoneal endometriosis. The results showed that RAC1 mRNA expression in peritoneal endometriosis significantly increased to 2.6 fold relative to normal endometrial $(\mathrm{p}=0.008)$ (Figure 1). While, the mRNA expression in peritoneal endometriosis was significant statistically compared to endometrial endometriosis $(\mathrm{P}=0.036)$ (Figure 1$)$. Furthermore, the RAC1 expression in endometrial endometriosis found moderate increased 1.3 fold relative to normal endometrial, but it was not significant statistically $(\mathrm{p}=0.303)$ (Figure 1$)$. 
Endometrial of women with endometriosis featured to be abnormal in cellular and molecular mechanism compared to unaffected women (Carvalho et al., 2011). Aberrant of endometrial tissues remodeling, adhesion or attachment, proliferation, and interaction with extracellular matrix and several MMPs are linked to the development of the diseases (Liu and Lang, 2011) It has been known that endometriosis is typically a disease-like cancer that has a metastatic process (Jiang et al., 2012). The metastatic process requires crosstalk between cell and extracellular matrix that linked to dynamic change of actin cytoskeleton. Thus, leading to focal adhesion formation (Jacquemet, 2015). RAC1, is one of focal adhesion genes, has been reported that increased its expression in endometriosis (Liu and Zhao, 2016). The results were also found in our study in the mRNA expression level of RAC1 gene. These results were similar to previous study that expression of RAC1 gene increased in endometrial endometriosis tissues (Ping et al.,2016). Zhao et al. (2009) also showed that focal adhesion genes underwent the upregulation of expression in peritoneal endometriosis tissue.

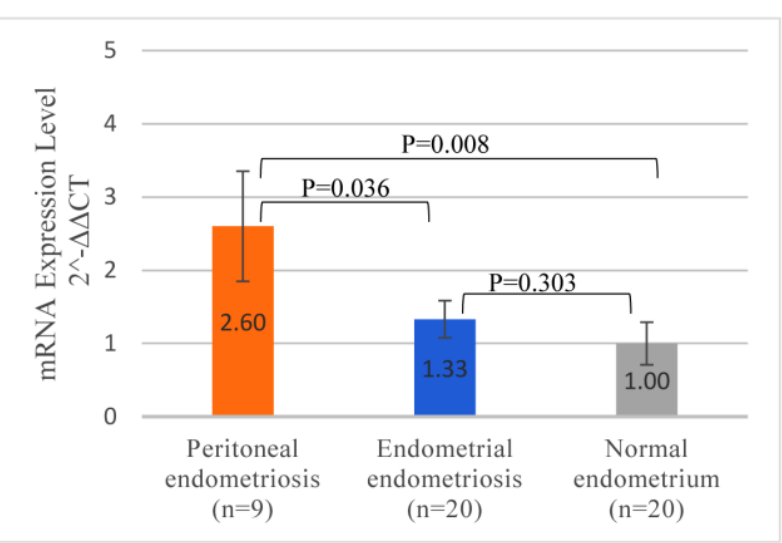

Figure 1. mRNA expression level of RAC1 gene in peritoneal and endometrial endometriosis as well as in normal endometrium.

\section{Promoter methylation level of RAC1 gene}

For DNA methylation profiling, we visualized products of MSP in gel electrophoresis from normal endometrium, endometrial and peritoneal endometriosis. The groups were divided into 3 categories of the band such as methylated, unmethylated, and partially methylated if the band appeared on both methylated and unmethylated. We also used $\mathrm{K}+$ containing only methylated primers and NTC (non- template control) containing only unmethylated primers. The $\mathrm{K}+$ and NTC were used to ensure the primers are not contaminated.

We collected endometriosis from two distinct tissues, which were 20 endometrial and 9 peritoneal tissues. From endometrial endometriosis, the results showed that 4 unmethylated, 1 methylated, and 15 partials methylated (Figure 2A), while 8 partial methylated and one unmethylated were shown from peritoneal tissues (Figure 2C). On the other hand, in control group that consisted of 20 samples of normal endometrium showed that 5 unmethylated, 1 methylated and 14 partials methylated (Figure 2B).

In this study we also quantified the percentage of promoter methylation of RAC1 gene in normal endometrium, endometrial and peritoneal endometriosis was $25.56 \%, 29.70 \%$, and $37.86 \%$, respectively. Promoter methylation level of RAC1 gene in peritoneal endometriosis compared to normal endometrium shown significant statistically $(\mathrm{p}=0.044) \quad$ (Figure 3$)$; but its methylation level was no significantly difference observed in peritoneal compared to endometrial endometriosis $(p=0.119)$ (Figure 3). We also showed no significant changes between endometrial endometriosis compared to normal endometrium $(p=0.577)$ (Figure 3). A previous study conducted by Meyer et al. (2014) reported that the increasing of promoter methylation level in PR gene with methylated allele found in endometriosis lesions, but not in allele of normal endometrium. Other studies by Xue et al. (2007) resulted that hypomethylated in promotor region of SF-1 gene and ER- $\beta$ in lesions affect to the increase of estrogen and progesterone resistance.

\section{Correlation between promoter methylation level and $\mathrm{mRNA}$ expression level of RAC1 gene in endometrial endometriosis}

Regulation of gene expression requires various components including specific transcription factors that act as regulatory proteins to bind and interact with certain promoter regions (Xin et al., 2008). One of epigenetic modifications that is closely related and most often occurs in the process of gene expression is DNA methylation. According to this result, analysis correlation in endometrial endometriosis shown association between promoter hypermethylation level to 
mRNA expression level $(\mathrm{R}=0.014 ; \mathrm{p}=0.952)$ (Figure 4).

Unlike hypermethylation that usually promote gene silencing, in contrast this study demonstrated that promoter hypermethylation may enhance mRNA expression. Simultaneously, this result was also in parallel with Weng et al. (2015) shown that the increase of EGFR gene expression in gastric cancer was affected by methylation process in $\mathrm{CpG}$ promoter regions and hence leads to the increase of mRNA expression. However, possible explanation for these conditions is that methylation at the promoter close to the transcription start site (tss) triggers conformational changes from chromatin in this area so as to increase gene transcription (Weng et al., 2015). In addition, our suggested that other mechanisms such as histone modification and single nucleotide polymorphisms (SNP) in promoter region play a role in gene expression.

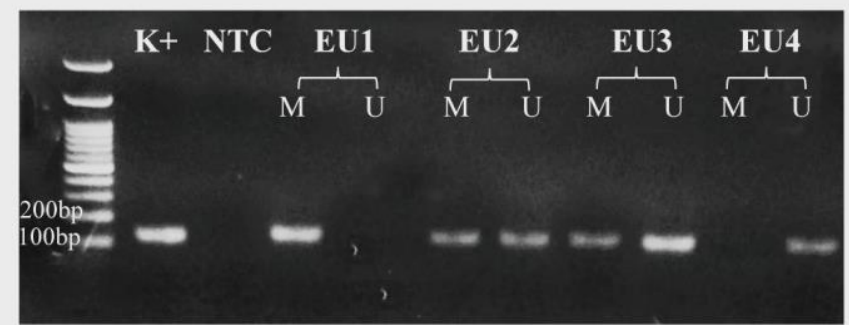

A

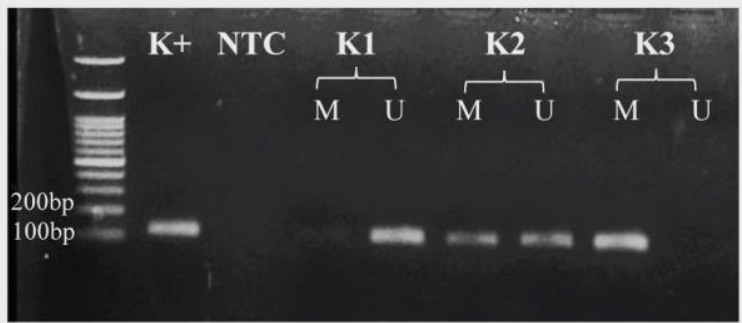

B

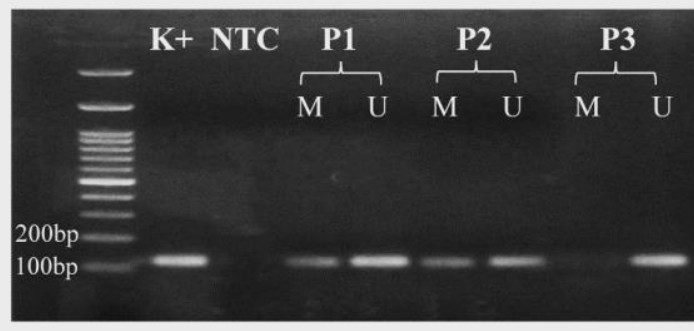

$\mathrm{C}$

Figure 2. Electrophoresis of MSP product for evaluation of promoter methylation level of RAC1 gene in different tissues. (A) in endometrial endometriosis (EU1-EU4); (B) in normal endometrium (K1-K3); (C) in peritoneal endometriosis $(\mathrm{P} 1-\mathrm{P} 3) . \mathrm{M}=$ methylated, $\mathrm{U}=$ unmethylated, $\mathrm{K}+=$ positive control, $\mathrm{NTC}=$ non template control.

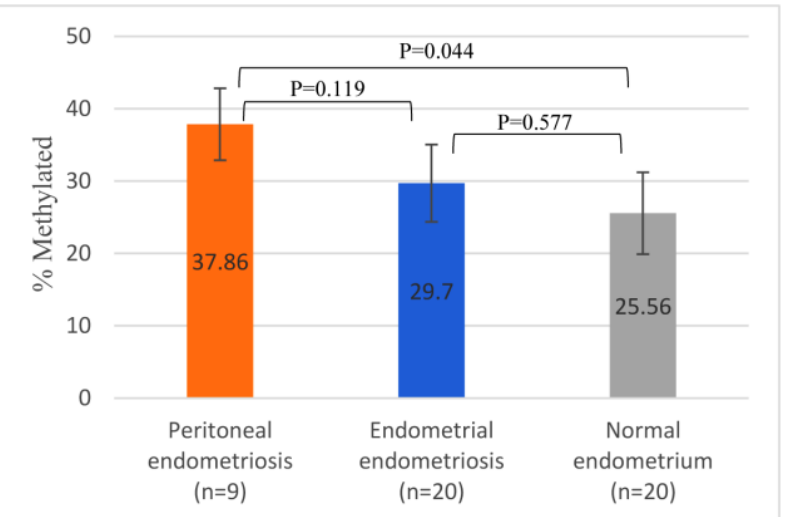

Figure 3. Promoter Methylation level of RAC1 gene in peritoneal endometriosis, endometrial endometriosis, and normal endometrium.

In endometriosis with the presence of endometrial-like tissues outside the uterine cavity is also termed as estrogen-dependent diseases
(Siva et al., 2014). Estrogen is required to promote tissues remodeling and endometrial cell migration by increasing focal adhesion formation and cell membrane morphology alteration (Flamini et al., 2011). This condition triggered protrusion of membrane such as lamellipodia and filopodia which facilitate cell migration (Flamini et al., 2011). Cell migration process is exemplified as in the study of Huang et al. (2016) proposes a hypothetical model of breast cancer that activated RAC1 promote cell migration and invasion (Huang et al., 2016). RAC1 gene has the activity to regulate and coordinate in assembly and disassembly process of the actin cytoskeleton (Faix \& Weber 2013).

RAC1 gene encodes a protein that is essential for normal cellular activities including phagocytosis, mesenchymal migration, axonal 
growth, and differentiation of several types cell (Bid et al., 2013). RAC1 appears to be deregulated in both expression and activity in a variety of tumor cells (Rathinam et al., 2011). RAC1 hyperactivation and overexpression seem closely related to aggressive growth and its activity is important in the processes that underlie malignant characteristic in several different tumor types (Rathinam et al., 2011).

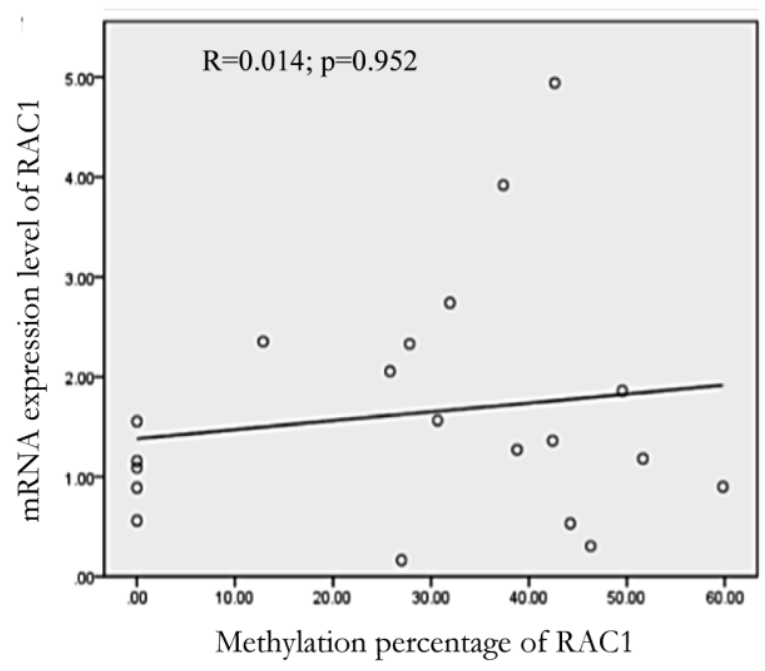

Figure 4. Correlation between promoter methylation level of RAC1 gene and mRNA expression level in endometrial endometriosis.

Overexpression of RAC1 gene led to the pathological condition and it can be found in endometriosis-associated epigenetic mechanisms. The epigenetic process plays a role in regulating gene expression may alter malignancy-associated characteristics. Alteration of methylation on DNA promoter is one of mechanisms related to changes in gene expression observed in endometriosis. However, it is difficult to determine whether alteration in methylation is the cause or consequences of this disease, considering that endometriosis is a multifactorial disease which can be caused by interactions between epigenetics and extrinsic factors.

DNA methylation is a reversible and is influenced by environmental factors, i.e. xenobiotic exposure, social behavior, metabolism and nutritional deficiencies that can have an effect later on, during a period of life development or can be brought down trans generatively (Koukora et al., 2014). In fact, Sofo et al reported that there was a correlation of dioxins with epigenetic changes, one of which is DNA methylation that occurs in endometriosis subjects. Exposure to dioxins is related to changes in gene expression associated with transcription regulation, proliferation, steroid sex metabolism, apoptosis, immune response and cell adhesion.

Since endometriosis is a multifactorial disease, reactive oxygen species (ROS) has been linked to epigenetic alteration in DNA methylation and histone modifications. ROS alters the epigenetic systems through eliminating the DNA and histone methylation marks as well as trigger in changing methylation specific site via regulation of DNMT expression and it will affect to the gene expression (Ito et al., 2017). Thus, it may be concluded that DNA methylation is not a single factor influenced the changes of mRNA expression, but other factors may be contributing to affect it. Based on this research, our future direction is to find a diagnostic biomarker to better understand the pathomechanisms of endometriosis through DNA methylation analysis and perform focal adhesion analysis and cell migration assay to evaluate the RAC1 protein that encourages migration cells in endometriosis.

\section{CONCLUSIONS}

An increase of RAC1 mRNA expression play a role in endometrial cell migration to peritoneum and promoter hypermethylation alteration of RAC1 gene associated with expression level of its gene.

\section{ACKNOWLEDGEMENTS}

We are thankful to Dimas Noor Ramadhian for English correcting.

\section{REFERENCES}

Acosta, C. L., Fallin, M. D. 2016. The role of epigenetics in genetic and environmental epidemiology. Epigenomics 8(2): 271-283. Aznaurova, Y. B., Zhumataev, M. B., Roberts, T. K., Aliper, A. M., Zhavoronkov, A. A. 2014. Molecular aspects of development and regulation of endometriosis. Reproductive Biology and Endocrinology 12(50): 1-25. 
Barcz, E., Milewski, Q., Dziunycz, P., Kaminski, P., PIoski, R., Malejczyk, J. 2012. Peritoneal cytokines and adhesion formation in endometriosis: an inverse association with vascular endothelial growth factor concentration. Fertility and Sterility 97(6): 1380-1386.

Bid, H. K., Roberts, R. D., Manchanda, P. K., Houghton, P. J. 2013. RAC1: An emerging therapeutic option for targeting cancer angiogenesis and metastasis. Molecular Cancer Therapentics 12: 1925-1934.

Burney, R. O., Guidice, L. C. 2012. Pathogenesis and pathophysiology of endometriosis. American Society for Reproductive Medicine 98(3): 511-519.

Carvalho, L., Podgaec, S., Bellodi-Privato, M., Falcone, T., Abrao, M. S. 2011. Role of eutopic endometrium in pelvic endometriosis. Journal of Minimally Invasive Gynecology 18(4): 419-427.

Darmawi, Marwali, M. L. S., Febri, R. R., Muharam, R., \& Hestiantoro, A. 2018. DNA methylation of the progesterone receptor B (PR-B) gene promoter in human eutopic endometrium, ectopic peritoneum, and ovarian endometriosis. Journal of Physics: Conference Series 1073: 1-16.

Dorak, T. 2006. Relative quantification. In: Real-Time PCR. Ed. Pfaffl, M.W. pp.63-66. Abingdon: Taylor \& Francis Group.

Faix, J., Weber, I. 2013. A dual role model for active RAC1 in cell migration. Small GTPases 24(2): 110-115.

Fassbender, A., Rahmioglu, N., Vitonis, A. F., Viganò, P., Giudice, L. C., D’Hooghe, T. M., Hummelshoj, L., Adamson, G. D., Becker, C. M., Missmer, S. A., \& Zondervan, K. T. 2014. World endometriosis research foundation endometriosis phenome and biobanking harmonisation project: IV. Tissue collection, processing, and storage in endometriosis research. Fertility and Sterility 102: 1244-1253.

Flamini, M. I., Sanchez, A. M., Genazzani, A. R., Simoncini, T. 2011. Estrogen regulates endometrial cell cytoskeletal remodelling and motility via focal adhesion kinase. Fertil Steril 95(2): 722-726.

Gupta, S., Harlev, A., \& Agarwal, A. 2015. Endometriosis A comprehensive update. NewYork: Springer.

Haller, B. W. 2012. Structure and function of focal adhesions. Current Opinion in Cell Biology 24: 116-124.

Huang, C., Verhulst, S., Shen, Y., Bu, Y., Cao, Y., He, Y., Wang, Y., Huang, D., Cai, C., Rao, K., \& Liao, D. F. 2016. AKR1B10 promotes breast cancer metastasis through integrin $\alpha 5 / \delta$-catenin mediated FAK/Src/RAC1 signaling pathway. Oncotarget 7(28): 1-11.

Ito, F., Yamada, Y., Shigemitsu, A., Akinishi, M., Kaniwa, H., Miyake, R., Yamanaka, S., \& Kobayashi, H. 2017. Role of oxidative stress in epigenetic modification in endometriosis. Reproductive Sciences 24(11): 1493-1502.

Jacquemet, G., Hamidi, H., \& Ivaska, J. 2015. Filopodia in cell adhesion, 3D migration, and cancer cell invasion. Current Opinion in Cell Biology 36: 23-31.

Jiang, Q. Y., Wu, R. J. 2012. Growth mechanisms of endometriotic cells in implanted places: a review. Gynecology Endocrinology 28: 562-567.

Koukoura, O., Sifakis, S., \& Spandidos, D. A. 2016. DNA methylation in endometriosis (Review). Molecular Medicine Reports 13: 2939-2948.

Liu, H., Lang, J. H. 2011. Is abnormal eutopic endometrium the cause of endometriosis? The role of eutopic endometrium in pathogenesis of endometriosis. Medical Science Monitor 17(4): 92-99.

Liu, L. J., Zhao, M. 2016. A PubMed-wide study of endometriosis. Genomics 108: 151-157.

Meyer, J. L., Zimbardi, D., Podgaec, S., Amorim, R. L., Abrão, M. S., \& Rainho, C. A. 2014. DNA methylation patterns of steroid receptor genes ESR1, ESR2 and PGR in deep endometriosis compromising the rectum. International
Journal of Molecular Medicine 33(4): 897-904.

Nagano, M., Hoshino, D., Koshikawa, N., Akizawa, T., Seiki, M. 2012. Turnover of focal adhesions and cancer cell migration. International Journal of Cell Biology: 1-10.

Ping, S., Ma, S., Liu, P., Yang, L., Yang, X., Wu, Q., Zhao, X., \& Gong, B. 2016. Molecular mechanisms underlying endometriosis pathogenesis revealed by bioinformatics analysis of microarray data. Archives Gynecology Obstetrics 293: 797-804.

Rathinam, R., Berrier, A., \& Alahari, S. K. 2011. Role of Rho GTPases and their regulators In cancer progression. Frontiers in Bioscience 16: 2561-2571.

Schübeler, D. 2015. Function and information content of DNA methylation. Nature 517: 321-326.

Siva., Srivastava, P., \& Shivaji, S. 2014. Understanding the pathogenesis of endometriosis through proteomics: Recent advances and future prospects. Proteomics Clinical Applications 8:86-98.

Sofo., Götte, M., Lagana, A. S., Salmeri, F. M., Triolo, O., Sturlese, E., Retto, G., Alfa, M., Granese, R., \& Abrao, M. S. 2014. Correlation between dioxin and endometriosis: an epigenetic route to unravel the pathogenesis of the disease. Archives of Gynecology and Obstetrics 292: 973-986.

Soo, H.A., Vinay, S., \& Chandrakant, T. 2017. Biomarkers in endometriosis: challenges and opportunities. American Society for Reproductive Medicine 107(3): 523-532.

Sourial, S., Tempest, N., Hapangama, D. K. 2014. Theories on the Pathogenesis of Endometriosis. International Journal of Reproductive Medicine: 1-9.

Weng, X., Zhang, H., Ye, J., Kan, M., Liu, F., Wang, T., Deng, J., Tan, Y., He, L., \& Liu, Y. 2015. Hypermethylated Epidermal growth factor receptor (EGFR) promoter is associated with gastric cancer. Scientific Reports 5: 1-10.

Xin, D., Hu, L., \& Kong, X. 2008. Alternative promoters influence alternative splicing at the genomic Level. PLoS One 3(6): 18.

Xue, Q., Lin, Z., Yin, P., Milad, M. P., Cheng, Y. H., Confino, E., Reierstad, S., \& Bulun, S. E. 2007. Transcriptional activation of steroidogenic factor- 1 by hypomethylation of the 5' CpG island in endometriosis. Journal of Clinical Endocrinology and Metabolism 92(8): 3261-3267.

Zhao Wang, Q., Bai, C., He, K., Pan, Y. 2009. A cross study gene set enrichment analysis identifies critical pathways in endometriosis. Reproductive Biology Endocrinology 7(94): 1-9. 